\title{
Comunicación

\section{Postgrados e investigación en comunicación en México: ¿estancamiento o evolución de la desarticulación múltiple?}

Graduate programs and communication research in Mexico: A stagnant or evolving multiple disarticulation?

RAÚL FUENTES NAVARRO 1

Se busca identificar e interpretar las tendencias más recientes de desarrollo de la investigación académica y los programas de postgrado en comunicación en México. Se sitúan los datos de referencia en los contextos que permitan discutir si se puede reafirmar la caracterización de este campo académico como sujeto a una desarticulación múltiple, en condiciones de "estancamiento concentrado" o de evolución "postdisciplinaria".

PALABRAS CLAVE: Comunicación, investigación, programas de postgrado, políticas nacionales, institucionalización.
This article seeks to identify and interpret the latest trends of development of scholarly research and graduate programs in communication in Mexico. Reference data are placed within the contexts best suited to discuss whether the characterization of this academic field as subject to multiple disarticulation is also subject to conditions of "concentrated stagnation" or "post-disciplinary" evolution.

KEY WORDS: Communication, research, graduate programs, national policies, institutionalization.

1 Instituto Tecnológico y de Estudios Superiores de Occidente y Universidad de Guadalajara, México.

Correo electrónico: raul@iteso.mx

Periférico Sur Manuel Gómez Morín 8585, C.P. 45604; Tlaquepaque, Jalisco, México. 
El campo científico es un espacio de intercambio dialógico entre investigadores y como tal un ámbito de la comunicación discursiva. Las

distintas teorías son enunciados producidos por alguien y dirigidos a alguien en un momento histórico determinado que responden de manera mediata o inmediata a enunciados previos. En ese sentido, parafraseando a Bajtín podemos decir que cada teoría es "un eslabón en la cadena de la comunicación discursiva” (De la Peza, 2013, p.15).

El campo avanza de la misma manera en que avanza una conversación, lo cual no es necesariamente decir que se mueva hacia algún ideal definido o hacia algún destino terminal. Al incorporarse nuevas voces a la conversación, al decirse cosas y responderse, se desarrolla la conversación y acumula una historia compartida que se convierte en punto de referencia (Robert T. Craig citado en Boromisza-Habashi, 2013, p. 421).

En los años más recientes, a diferencia de épocas pasadas, los estudios de comunicación aparecen ya, con naturalidad y dignidad, en muchos de los recuentos y revisiones como los incluidos en el Informe sobre las Ciencias Sociales en el mundo 2010 (Organización de las Naciones Unidas para la Educación, la Ciencia y la Cultura [UNESCO]-Consejo Internacional de Ciencias Sociales [CICS], 2012), especialmente en referencia a los procesos de transformación de los "territorios disciplinarios", cuando se hace notar, por ejemplo, que:

Los estudios de la comunicación son un campo de investigación relativamente nuevo. Tienen algunos de los rasgos de un campo transdisciplinario e interdisciplinario; sin embargo, recientemente han adquirido mucha de la parafernalia institucional y profesional de una disciplina académica, incluyendo crecientes ofertas de cursos universitarios, lo cual resulta en un número mayor de académicos contratados, departamentos en universidades, asociaciones profesionales nuevas y conferencias. Actualmente "comunicación" es identificada como una categoría separada en las bases de datos bibliográficas de ciencias sociales como el SSCI Thomson Reuters, y el número de artículos publicados en esta categoría muestra una tendencia a aumentar. Incluso esto puede no reflejar el número aún mayor de libros de texto publicados anualmente en este campo (p. 204). 
Una pregunta que el propio Informe... formula en relación con los cruces de las fronteras disciplinarias en ciencias sociales podría ser, quizá, mejor respondida atendiendo al desarrollo de los estudios de comunicación y a casos aparentemente atípicos, como el mexicano: “¿Cómo puede ser fortalecida la formación interdisciplinaria mientras que las disciplinas se fortalecen? Esta puede ser la pregunta práctica de mañana para la investigación en ciencias sociales" (UNESCO-CICS, 2012 , p. 213). Desde la región iberoamericana, voces autorizadas en los estudios de comunicación, como la de Miquel De Moragas (2011), refuerzan esta perspectiva:

La historia de la investigación sobre la comunicación nos pone de manifiesto que los planteamientos que han pretendido constituir una 'disciplina independiente', para obtener reconocimiento y prestigio académicos, han resultado ser muy poco rentables en términos de desarrollo de nuestros conocimientos sobre la comunicación (p.19).

Al considerar dichos estudios al mismo tiempo, aunque desde distintos ángulos, como un campo y una disciplina, para De Moragas (2011) es indispensable reconocer cómo "en cada época histórica, en cada país y en cada región, la investigación recibe demandas sociales distintas, dependientes de los centros de decisión política, económica y cultural" (p.15). Lo mismo puede atestiguarse, obviamente, respecto de los programas de postgrado en que se forman los nuevos investigadores. Junto a las asociaciones académicas y las publicaciones, los programas universitarios son objetivaciones primarias y por lo tanto, evidencias empíricas concretas de los procesos de institucionalización de los campos y disciplinas académicos.

A pesar de la mundialización (Mattelart, 1998, 2006), "las diferencias entre regiones y países en el estatus de la investigación de las ciencias sociales no podrían ser mayores" (UNESCO-CICS, 2012, p. 53). Datos de la década pasada indican que:

El 90 por ciento de las instituciones de educación superior en América Latina no producen ningún tipo de investigación, mientras que más de dos tercios de todos los programas de postgrado se ofrecen en las universidades públicas 
de Brasil y México, y ahí es donde se efectúa la mayoría de las investigaciones (p. 53).

De la misma manera que los procesos de institucionalización de los estudios de comunicación presentan amplísimas diferencias entre países que se reflejan en los grados de consolidación académica de los programas de postgrado, así como en la contribución de cada país a la investigación internacional, ${ }^{2}$ persisten también, al interior de los países, brechas y polarizaciones crecientes en términos regionales y sectoriales que las políticas de educación superior y de ciencia y tecnología de los estados nacionales enfrentan con diversos grados de eficacia y pertinencia. La internacionalización desigual, constatable entre los países iberoamericanos, afecta también a la región en su conjunto comparada con otras regiones geográficas y lingüísticas del mundo, situación que ha motivado la constitución de una Confederación Iberoamericana de Asociaciones Científicas y Académicas de Comunicación (CONFIBERCOM), cuya misión puede resumirse en construir y transitar continuamente los puentes internacionales, interdisciplinarios e interinstitucionales para mejorar la calidad y la diversificación de la enseñanza y la investigación en esta área de estudios y el fomento de iniciativas para estimular la cooperación entre instituciones, países y regiones de Iberoamérica. ${ }^{3}$

2 No obstante que el español y el portugués son respectivamente la cuarta y la quinta lenguas más usadas en el mundo entre las revistas indizadas en ciencias sociales, según indicadores de Ulrich y de Thomson-SSCI, siendo superadas solamente por el inglés, el francés y el alemán, la proporción de la producción mundial que difunden es mínima, pues en conjunto alcanzan apenas entre 0.5 y $5.7 \%$ mundial, dependiendo de los periodos y los métodos de medición (UNESCO-CICS, 2012).

3 Entre las principales perspectivas intelectuales que han confluido en esta iniciativa, pueden destacarse la del brasileño José Marques de Melo (2012) y la del español Francisco Sierra Caballero (2013). Lo más novedoso, probablemente, es la inclusión de España y Portugal junto a los países latinoamericanos en una entidad mayor, denominada Iberoamérica, en la que sin embargo no caben otros países latinoeuropeos, como Francia, Italia o Rumania. 
Una de las maneras previstas por los promotores de esta organización para avanzar en la realización de sus propósitos han sido sus foros, un modelo de cooperación internacional basado en el principio de autonomía y coordinación permanente, centrado en la construcción de espacios de reflexión, análisis y articulación de políticas públicas en el campo que atañe a los estudios superiores en comunicación en Iberoamérica, específicamente sobre los sistemas nacionales de apoyo a la ciencia y la tecnología, los programas de postgrado y las publicaciones. Después de participar en las tres primeras ediciones de estos foros, ${ }^{4}$ el autor de este artículo pretende sintetizar y exponer aquí los principales avances alcanzados desde su particular punto de vista y recontextualizarlos en relación con los marcos institucionales mexicanos y los análisis que pueden considerarse antecedentes, así como las perspectivas y tendencias de desarrollo que se pueden identificar en el país.

\section{CONTEXTOS, PERSPECTIVAS Y TENDENCIAS DEL POSTGRADO EN COMUNICACIÓN EN MÉXICO}

Al igual que el desarrollo institucional general de los estudios sobre comunicación en las universidades latinoamericanas (Federación Latinoamericana de Facultades de Comunicación Social [FELAFACS], 2009), la investigación y el postgrado en esta especialidad presentan tal diversidad que es difícil atribuirle características nacionales, y con mayor razón, megaregionales (latinoamericanas o iberoamericanas). 5 El alcance de las políticas gubernamentales para fomentar, evaluar y reconocer los progresos y articulaciones de los programas es desigual $\mathrm{y}$, en ciertos sentidos, contradictorio. Distintos proyectos recientes han

4 Celebrados en São Paulo, Brasil en agosto de 2011; en Quito, Ecuador en abril de 2012, y en Maia, Portugal, en mayo de 2013.

5 Significativamente, los informes iniciales generados por la CONFIBERCOM sobre los postgrados en comunicación en nueve países de la subregión sur (Cimadevilla \& García Corredor, 2012) y otros nueve de la subregión norte (Fuentes Navarro \& Bustamante Farias, 2012) de América Latina, fueron subtitulados respectivamente "heterogeneidades entrelazadas" y "una expansión asimétrica y desarticulada”. 
aportado valiosos elementos de interpretación de esa diversidad respecto del desarrollo de la ciencia y la tecnología (Albornoz, 2010), las ciencias sociales en América Latina (Trinidade, 2007) o la formación de postgrado (Luchilo, 2010a), aunque no puede hablarse de un marco compartido dentro del cual construir un diagnóstico y una estrategia iberoamericanos en función del postgrado en comunicación (Vassallo de Lopes, 2012). Cruz Cardona y Martos Perales (2010) advierten que:

Aunque pueden encontrarse algunas experiencias de evaluación de la calidad de los programas de formación superior avanzada, de casi 50 años de antigüedad en Brasil, y experiencias más o menos sistemáticas desde hace casi 20 en países como Argentina, Colombia, Cuba, España y México, considerada globalmente y aplicada de manera sistemática, la evaluación de la calidad del postgrado es un tema relativamente nuevo en el ámbito iberoamericano (p. 3).

Estos analistas (colombiano y español, respectivamente, directivos principales de la Asociación Universitaria Iberoamericana de Postgrado, [AUIP] ), ilustran la dificultad que genera la heterogeneidad existente en materia de educación superior en tamaño, y por ende en el número de egresados universitarios, así como en cuanto a su distribución en las distintas áreas de conocimiento, aunque en prácticamente todos los países predomina el área de ciencias sociales y administrativas (dentro de la cual se ubican los estudios de comunicación), sobre las restantes: ciencias de la salud y los servicios, ciencias exactas e ingenierías, y humanidades y artes, apuntando respecto de la evaluación de la calidad de los postgrados que:

Siete años después de que la AUIP publicara un estudio sobre gestión de la calidad del postgrado en Iberoamérica, la situación no ha cambiado mucho en cuanto a los paradigmas y modelos de evaluación más comúnmente utilizados. Los propósitos apenas se han reformulado y, de ellos, sobresalen, particularmente en Argentina, Brasil, Colombia, Cuba, España y México, los siguientes: mejorar la calidad; crear y fortalecer una cultura de evaluación para rendición de cuentas y someterse a procesos de aseguramiento, reconocimiento o acreditación de la calidad (Cruz Cardona y Martos Perales, 2010, p. 6). 
Durante el Foro Iberoamericano de Postgrado en Comunicación celebrado en São Paulo en agosto de 2011 como parte del I Congreso Mundial de Comunicación Iberoamericana de la CONFIBERCOM (Kunsch \& Marques de Melo, 2012) y en su secuela realizada en Quito en abril de 2012 (Kunsch, 2013), se presentaron los informes de cinco comisiones subregionales encargadas de hacer un recuento actualizado de los programas de postgrado en comunicación existentes en Iberoamérica, ${ }^{6}$ que nadie más había levantado en más de una década, los cuales se reunieron en una publicación digital (Vassallo de Lopes, 2012) sin que hasta principios de 2014 se haya intentado analizarlos o interpretarlos en conjunto.

No obstante, la agregación de la información generada dejó claras, al menos, tres condiciones: a) el notable crecimiento de la oferta de postgrado en comunicación, pues el inventario sistematizado rebasó los 450 programas (maestrías y doctorados) en total, situados en 21 países, una alta proporción de ellos fundados en la última década (véase Tabla 1); b) la dificultad de homologar los sistemas nacionales que regulan la creación, el desarrollo y la evaluación de estos programas, a pesar de las coincidencias detectadas en el nivel de las políticas, ${ }^{7}$ y c) la opor-

6 Estos equipos de trabajo estuvieron integrados por Maria Immacolata Vassallo de Lopes y Richard Romancini (Universidad de São Paulo) subregión Brasil; Antonio García Jiménez (Universidad Rey Juan Carlos) y Josep Lluís Gómez Mompart (Universidad de Valencia) subregión España; Moisés De Lemos Martins y Madalena Oliveira (Asociación Portuguesa de Ciencias de la Comunicación) subregión Portugal; Raúl Fuentes Navarro y Óscar Bustamante (Instituto Tecnológico y de Estudios Superiores de Occidente) subregión México, Centroamérica y Caribe, y "Colectivo Sur", coordinado por Gustavo Cimadevilla (Universidad Nacional de Río Cuarto) y Claudia G. Corredor (Universidad Javeriana) subregión Sudamérica, exceptuando Brasil.

7 Un ejemplo elocuente es que a pesar de que los sistemas oficiales de evaluación del postgrado en México y Brasil son prácticamente iguales, en Brasil son de observación obligatoria y en México opcionales. En Brasil, en consecuencia, un programa que no adquiere en un plazo determinado las calificaciones mínimas de acreditación o las pierde, debe desaparecer, 
tunidad de consolidación de los estudios de comunicación como área académica que la cooperación internacional ofrece a las instituciones y asociaciones nacionales y regionales, mediante la articulación de esfuerzos y recursos invertidos en la formación universitaria de alto nivel (Vassallo de Lopes, 2012).

Según el "Mapa de la formación de comunicadores" elaborado por la FELAFACS para la UNESCO en 2009, la hipótesis que bien podría resumir el panorama formativo en la región, en el nivel de licenciatura (equivalente en algunos países al bacharelado, el grado o el pregrado), es la de una innovación de cara al mercado. Esto es, un proceso de adecuación por parte de las instituciones de educación superior (en distinto grado, pero todas, no solo las privadas) a las exigencias provenientes del ámbito laboral que hacen prevalecer un tipo de formación orientada a las tareas reproductoras (docencia), antes que a las prácticas de producción (investigación) de conocimiento relevante.

En el nivel de postgrado dos rasgos pueden ilustrar lo anterior: de un lado, la dedicación casi absoluta de la planta de profesores a la función docente y una reducción de las plazas laborales académicas de tiempo completo; de otro lado, los programas de postgrado, mayoritariamente maestrías, están orientados a un tipo de especialización profesional o de profesionalización docente como perfiles de egreso. En ambos casos, las tareas de investigación aparecen subordinadas al cumplimiento de estas exigencias, desaprovechando la oportunidad de retroalimentar con conocimiento nuevo la propia función docente. A esto se refiere Lucio (2002) cuando describe este tipo de cursos como postgrados de consumo. Surgen de ahí, al menos, dos cuestiones relevantes: en primer lugar, que pocas unidades académicas y programas de comunicación participan de procesos de acreditación, ya sea gubernamental, de agencias privadas o internacional en la mayoría de los países latinoamericanos, y muy notablemente en México. En segundo lugar, la paradoja de que al tiempo que se demandan profesores con formación de postgrado, hay pocos académicos de tiempo completo en las escuelas de comunicación y no se evidenciarían aportes de in-

mientras que en México hay espacio legítimo para la coexistencia de programas acreditados y no acreditados. 


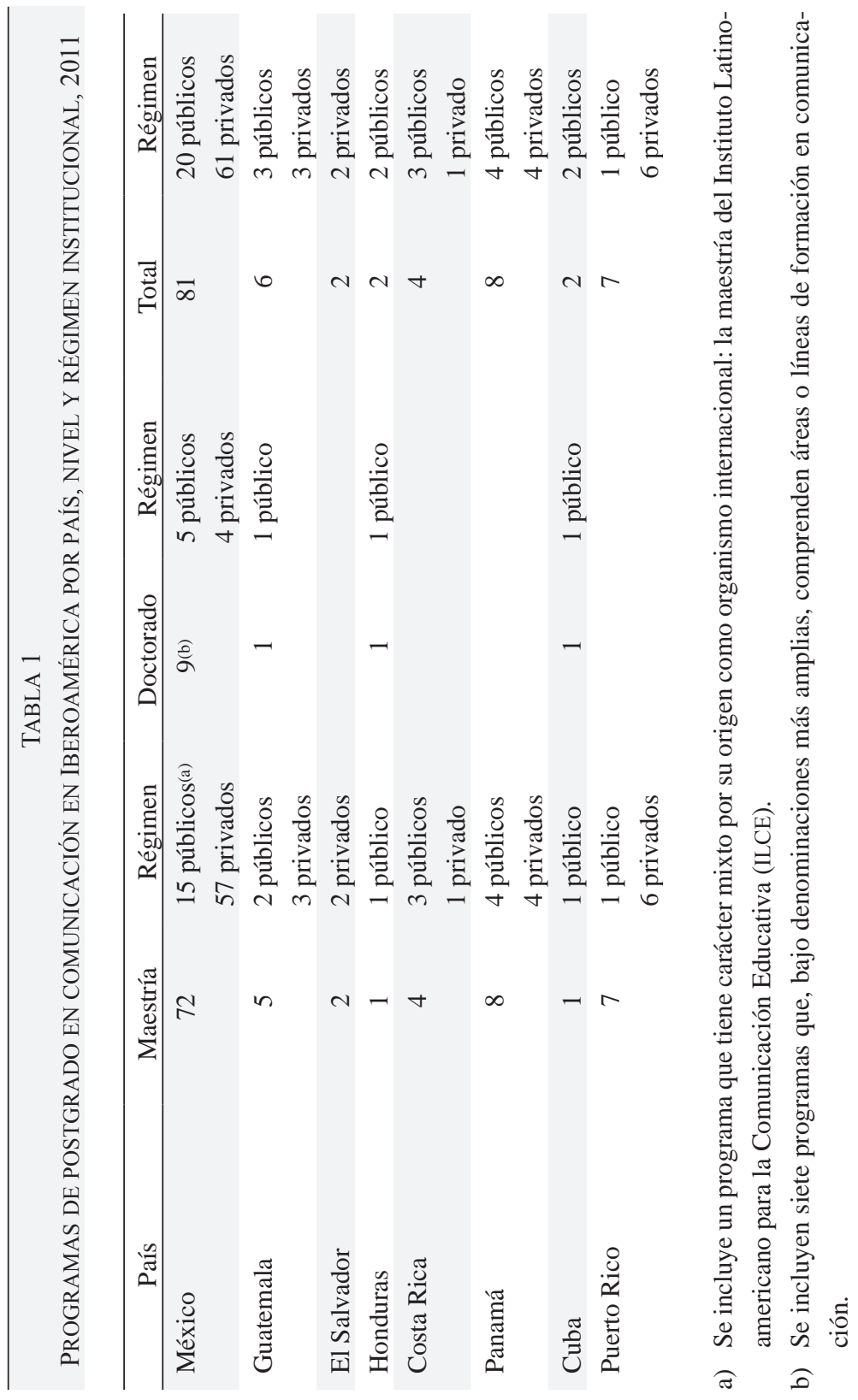




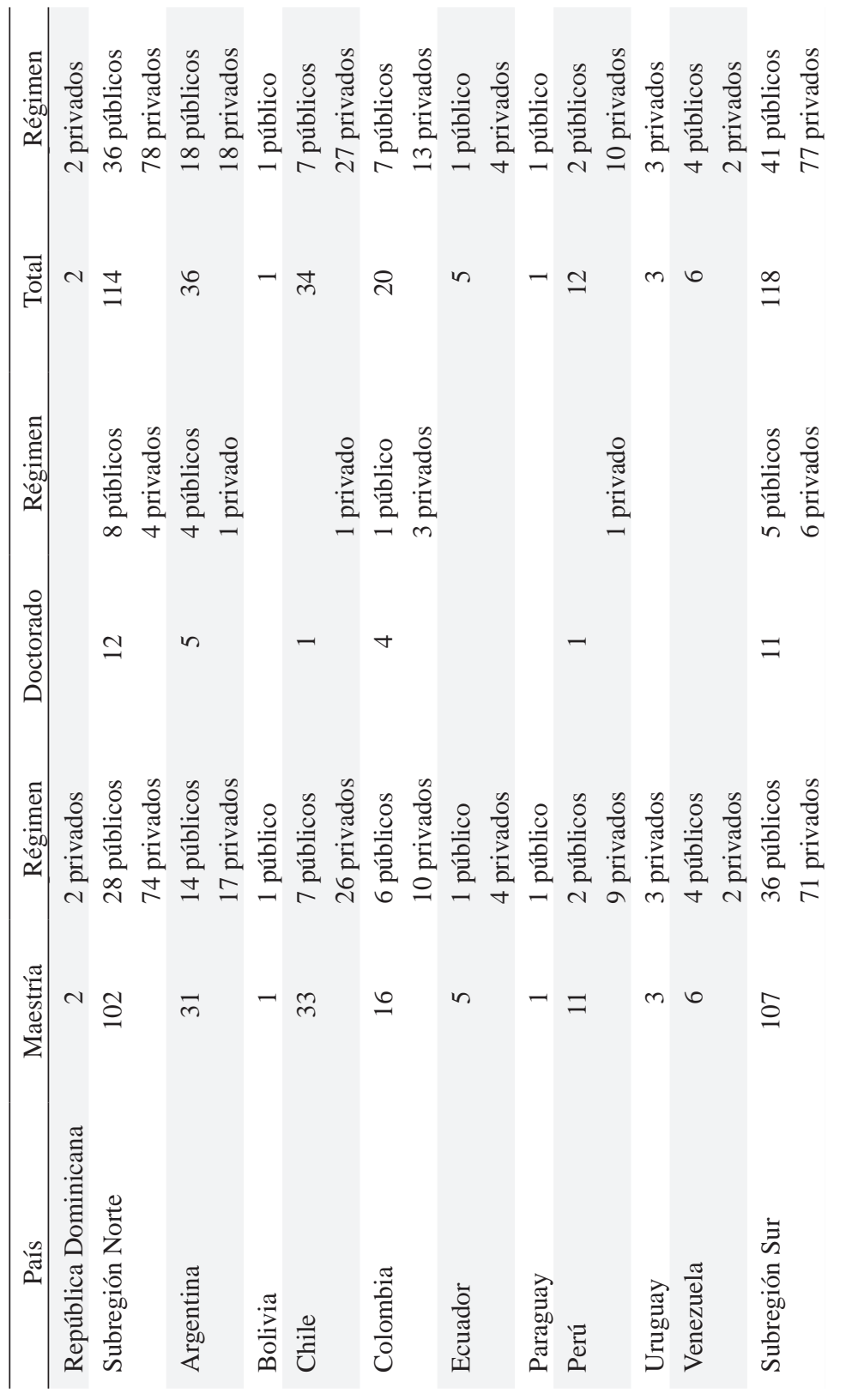




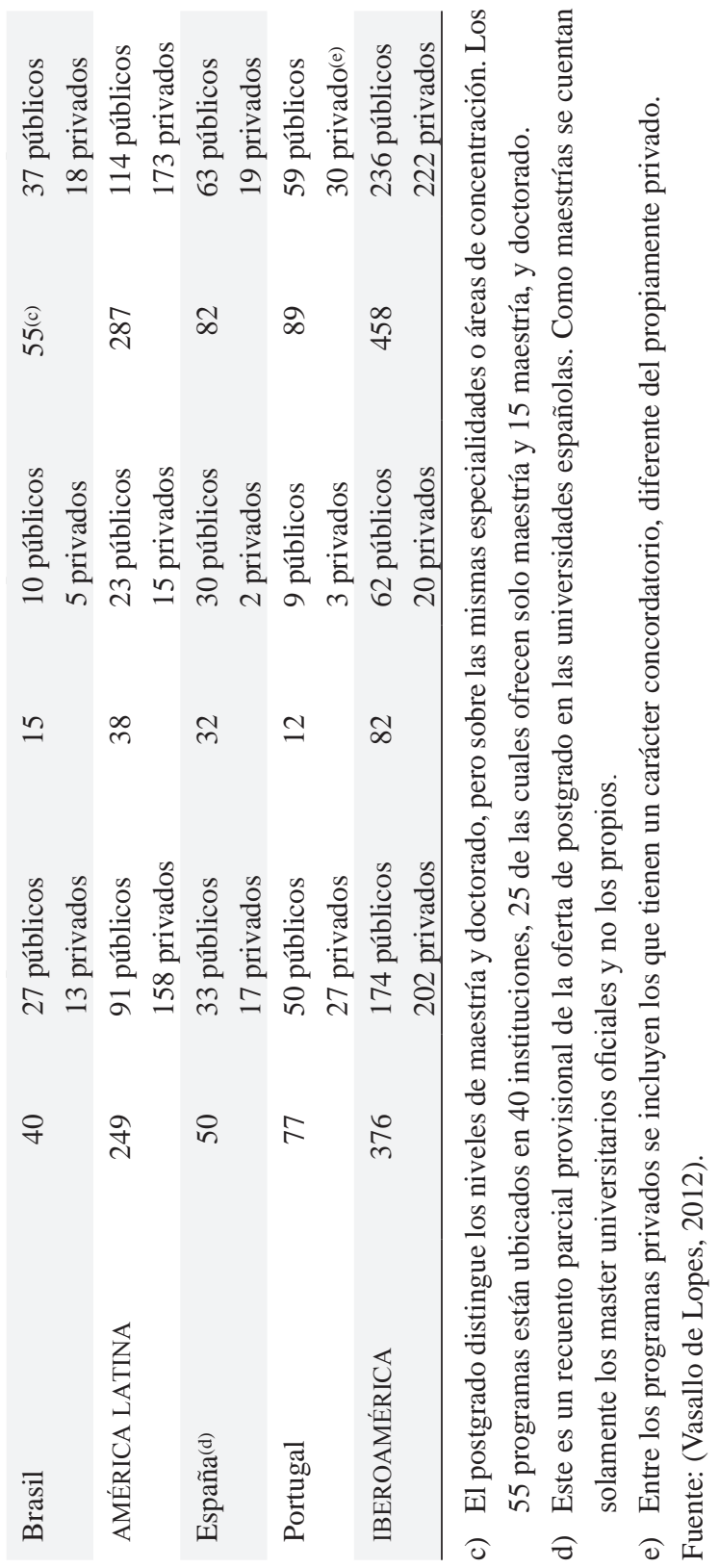


vestigación significativos en la mayoría. Las instituciones donde se ha institucionalizado la investigación y su articulación con la formación de postgrado pueden obtener la acreditación, especialmente de los programas más orientados hacia la formación de investigadores que hacia la profesionalización en otras especialidades, pues es indudable que la investigación académica requiere también de una profesionalización avanzada, lo cual permite replantear paradojas como la señalada. ${ }^{8}$

Aunque los primeros programas mexicanos de postgrado en comunicación fueron fundados en la década de los setenta (las maestrías de la Universidad Iberoamericana y la Universidad Nacional, ambas en la Ciudad de México), coincidiendo con los inicios de la institucionalización universitaria de la investigación en el campo (Fuentes Navarro, 2010, 2011), el desarrollo de este nivel ha sido precario a pesar de su crecimiento en la última década, y en su mayor parte dependiente de las mismas tendencias y condiciones de los programas de licenciatura, que han superado ya el millar en el país, según el recuento de Rebeil Corella (2009) para FELAFACS. ${ }^{9}$ Como sugiere este documento, "la proliferación

8 Aunque también hay que considerar que en la escala más general del sistema mexicano de educación superior, orientado histórica y prioritariamente a la formación de profesionales de cada vez más diversas especialidades mucho más que a la investigación, se conoce muy poco, al menos en un sentido sistemático y crítico, sobre la estructuración y la dinámica de las profesiones, sobre la inserción social de los egresados universitarios y sobre los factores que condicionan y caracterizan esa inserción. Aunque teóricamente hay algunos rasgos comunes entre las "disciplinas" y las "profesiones" (Abbott, 1988, 2001), casi toda la investigación disponible se refiere a realidades nacionales muy distintas a las de esta región, y es muy poco lo que se ha hecho en México para documentar la pertinencia de la producción universitaria propia, rasgo que es notable, aunque no exclusivo, respecto de los estudios de comunicación.

9 Si bien en el Anuario Estadístico de Educación Superior de la Asociación Nacional de Universidades e Instituciones de Educación Superior, fuente oficial mexicana de este tipo de información, se incluyen "solamente" 550 programas de licenciatura en comunicación (en sus diversas denomi- 
desmedida y sin control de las escuelas de comunicación marca una oferta de bajos estándares y que produce, en cantidad y en calidad, egresados que no pueden ser absorbidos por el mercado laboral", pues también se constata en esa proliferación el predominio abrumador de instituciones de "dudosa calidad" sobre "las universidades, escuelas y centros de gran tradición y calidad" (Rebeil Corella, 2009, p. 86).

Sin pretender actualizar o retomar por ahora el análisis de la oferta de licenciatura en comunicación (Fuentes Navarro, 2005), cabe únicamente constatar que para el ciclo escolar 2010-2011, la Asociación Nacional de Universidades e Instituciones de Educación Superior (ANUIES) registra una matrícula de 73513 estudiantes de ciencias de la comunicación, menos de $7 \%$ de los más de un millón de estudiantes del área de ciencias sociales y administrativas, y menos de 3\% del total de estudiantes de licenciatura universitaria y tecnológica (ANUIES, 2011). En 2003, la cifra registrada por la ANUIES fue de 64476 estudiantes de licenciatura en ciencias de la comunicación, lo que representaba 7.2\% de los estudiantes de ciencias sociales y administrativas y $3.5 \%$ de la matrícula total del nivel (Fuentes Navarro, 2005). Junto a esta disminución porcentual, el crecimiento de poco más de $12 \%$ en siete años del número de estudiantes de comunicación está claramente por debajo de $26 \%$ que creció la matrícula total del nivel en ese mismo periodo; se puede entonces reafirmar que no hay razones especiales de alarma por el crecimiento numérico de los programas o de los estudiantes, aunque sí por otras razones, referidas a la calidad de la oferta o de sus productos, o a la desproporción entre el nivel de licenciatura y el de postgrado.

En 2003, la ANUIES registraba una matrícula conjunta de apenas 957 estudiantes para 43 programas de maestría, con 18 denominaciones diferentes, correspondientes a "Ciencias de la Comunicación". En 2010-2011, la matrícula ascendió a 1354 estudiantes (casi 30\% de crecimiento) en 71 programas con 39 denominaciones diferentes. Y si se suman los programas clasificados bajo la categoría "Publicidad" (aunque no "Mercadotecnia") y otras menores, la matrícula llega a los

naciones), pertenecientes a 417 instituciones, en el ciclo escolar 2010-2011 (ANUIES, 2011). 
1662 estudiantes. De cualquier manera, la caracterización de este campo académico como sujeto a una hipertrofia en el nivel de licenciatura y al mismo tiempo a un claro subdesarrollo en el postgrado (Fuentes Navarro, 2005), mientras que ambos niveles tienden a la fragmentación, puede considerarse tan vigente como hace una década.

\section{LA EVALUACIÓN EN LOS SISTEMAS CIENTÍFICO Y DE EDUCACIÓN SUPERIOR EN MÉXICO}

En 2009, la presidenta en turno de la Academia Mexicana de Ciencias (AMC), Rosaura Ruiz Gutiérrez, sintetizó en un diagnóstico el contraste entre la consideración de que en el actual contexto, la educación superior, la ciencia, la tecnología y la innovación tienen importancia fundamental como instrumentos para "la construcción de sociedades del conocimiento, el desarrollo democrático y plural, contribuir al proceso de integración y el crecimiento económico, y la competitividad" por una parte, y por otra que "los resultados alcanzados dejan mucho que desear", pues la cobertura de cohorte de edad correspondiente a estudios superiores ha avanzado muy poco, se han profundizado las restricciones financieras, se han segmentado y diferenciado enormemente las instituciones, se ha mercantilizado su oferta y servicios, y no se ha podido concretar su articulación con el desarrollo del país (Ruiz Gutiérrez, 2009)10. Entre los datos oficiales que respaldan tal diagnóstico, incluidos en el Informe General del Estado de la Ciencia y la Tecnología, México 2009, publicado por el Consejo Nacional de Ciencia y Tecnología (CONACyT), se documenta que la Tasa Bruta de Cobertura de la Educación Superior es de 27\%; el Gasto Federal en Ciencia y Tecnología como proporción del Producto Interno Bruto (PIB) de $0.45 \%$, y que la oferta de educación superior incluye alrededor de 9000 programas de postgrado, entre ellos programas de doctorado en 200 instituciones de educación superior, 60\% de ellas públicas (CONACyT, 2010).

10 Datos incluidos en la presentación de la ponencia "Tendencias y perspectivas de la educación superior, la ciencia, la tecnología y la innovación en México" durante la Semana de la Ciencia y la Innovación 2009 (Boletín AMC/129/09). 
El marco legal fundamental de la evaluación de las prácticas científicas está dado en la Ley de Ciencia y Tecnología (promulgada en 2002), cuyo artículo 12 señala, entre los "Principios Orientadores de las actividades científicas", que "los resultados de las actividades de investigación, desarrollo tecnológico e innovación que sean objeto de apoyos en términos de esta Ley serán invariablemente evaluados y se tomarán en cuenta para el otorgamiento de apoyos posteriores" (Cámara de diputados, 2013). Además de la formalización del CONACyT como organismo rector del sector, dentro de su estructura orgánica la Ley estableció también el Sistema Nacional de Evaluación Científica y Tecnológica (SINECYT) como una instancia permanente de coordinación, cuyo "propósito fundamental es garantizar que la evaluación de las propuestas que se presentan en los diversos fondos de apoyo del CONACyT, se efectúe de manera transparente y objetiva", mediante la participación de los propios científicos en una estructura de siete Comisiones Dictaminadoras que representan grandes áreas del conocimiento, al interior de las cuales se especifican las "disciplinas" que las constituyen.11

El Sistema Nacional de Investigadores (SNI) fue creado en 1984, para reconocer las actividades de las personas dedicadas a producir conocimiento científico y tecnológico. De acuerdo con el artículo 54 de su Reglamento, las distinciones que confiere el SNI se clasifican en tres ca-

11 Área I. Físico-matemáticas y Ciencias de la Tierra (Astronomía, Ciencias de Materiales, Física, Geofísica, Geología, Matemáticas y Oceanografía); Área II. Biología y Química (Biología y Química); Área III. Medicina y Ciencias de la Salud (Ciencias de la Salud, Farmacia, Medicina y Odontología); Área IV. Humanidades y Ciencias de la Conducta (Arquitectura, Antropología, Bellas Artes, Biblioteconomía, Educación, Filosofía, Historia, Lingüística, Literatura y Psicología); Área V. Ciencias Sociales (Administración, Ciencia Política y Administración Pública, Comunicación, Contabilidad, Demografía, Derecho y Jurisprudencia, Economía, Geografía Humana, Sociología y Prospectiva); Área VI. Biotecnología y Ciencias Agropecuarias (Agronomía, Biotecnología, Medicina Veterinaria, Pesca y Zootecnia); Área VII. Ingenierías (Aeronáutica, Ambiental, Civil, de Comunicaciones, Electrónica y Control, Eléctrica, Computación, Industrial, de Materiales, Marina y Portuaria, Mecánica, Minera, Nuclear, Petrolera, Química y Textil). 
tegorías: I. Candidato a Investigador Nacional; II. Investigador Nacional, con tres niveles, y III. Investigador Nacional Emérito (CONACyT, 2008). La distribución de los 19747 miembros del SNI en 2013, por su categoría, indicaba $19 \%$ de candidatos, $54 \%$ de investigadores de nivel I; $18 \%$ de nivel II, y 9\% restante de nivel III (Foro Consultivo Científico y Tecnológico [FCCYT], 2013). Los requisitos mínimos que debe cubrir un investigador para ingresar al SNI son el grado de doctor y adscripción institucional para realizar actividades de investigación al menos por 20 horas/semana en alguna institución pública o privada. Para obtener y renovar la distinción, el investigador es evaluado "por pares", según el proceso que se resume en la Figura 1. En los últimos años, junto al crecimiento constante del número de miembros del SNI, se constata una disminución en el "Coeficiente de aprobación" o porcentaje de solicitudes aprobadas, que en la última década pasó de cerca de $80 \%$ a alrededor de $65 \%$.

La definición de las disciplinas y especialidades de investigación determinante para la evaluación depende principalmente de la autoadscripción de los investigadores al presentar su solicitud. Si bien hay algunos investigadores del campo de la comunicación que pertenecen al SNI desde los primeros años posteriores a su creación en 1984, apenas en la última década pudieron identificarse como tales, pues la categoría comunicación como disciplina de las Ciencias Sociales no estaba incluida entre las originales. El propio crecimiento del número de investigadores autodefinidos como de comunicación es la causa de su reconocimiento bajo una categoría disciplinaria específica, y éste provocó que, a partir de 2006, se incluyera a un investigador de la comunicación entre los miembros de la comisión Dictaminadora de Ciencias Sociales (Área V). No obstante, todavía hay un porcentaje de académicos cuyo trabajo es reconocido en el campo de la comunicación que prefieren ser evaluados bajo otra categoría disciplinaria, incluyendo las adscritas al Área de Humanidades y Ciencias de la Conducta. El número de investigadores de la comunicación en el SNI ha crecido de una manera muy rápida en la última década, y alcanza ya aproximadamente 1\% de los miembros totales del Sistema (193 en 2014) pero su distribución por categorías es distinta del total del SNI: 7\% de candidatos, $65 \%$ de nivel I, $17 \%$ de nivel II y $11 \%$ de nivel III.

Paralelamente, el crecimiento del postgrado en el sistema mexicano de educación superior presenta algunas características en común y cier- 


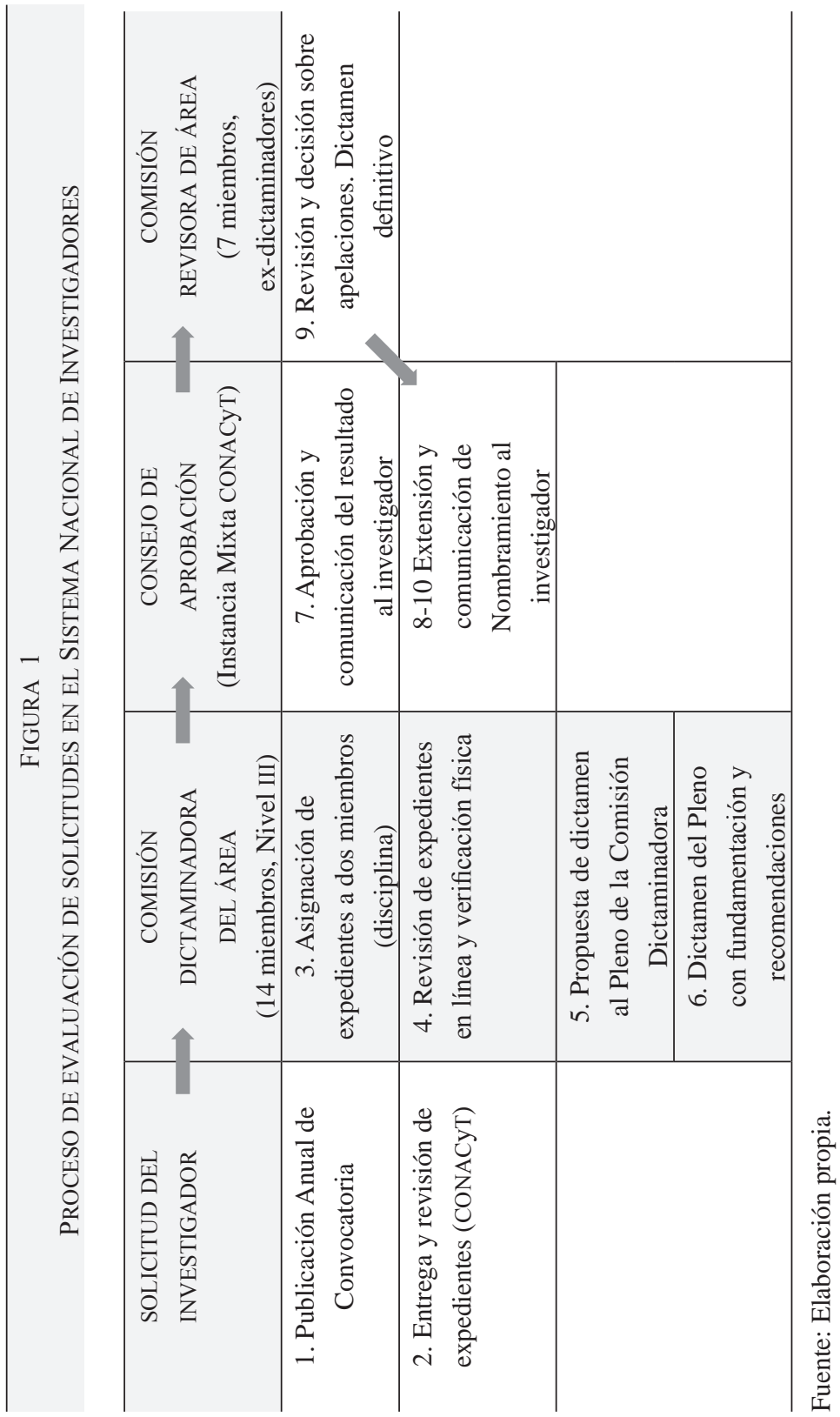


tas diferencias con los de otros países latinoamericanos. Una síntesis de las tendencias recientes en la formación de postgrado señala que:

En la década de 1960 creció un $87 \%$ y en la de 1970 un 215\%. Si bien en las décadas siguientes las tasas de crecimiento fueron menores $-46 \%$ en la de 1980 y 64\% en la de 1990-, el resultado final ha sido de una expansión muy importante (Luchilo, 2010b, p. 180). ${ }^{12}$

Una de las consecuencias más directas de esta expansión ha sido la insuficiente formación de profesores: "Hacia 1970, había alrededor de 10000 profesores ocupados en instituciones de educación superior; treinta años más tarde, esa cantidad se había multiplicado hasta alcanzar los 208.000 profesores" (Luchilo, 2010b, p. 179). En el sistema mexicano de educación superior en su conjunto, la expansión se ha sustentado en la creación de numerosas instituciones nuevas, especialmente de carácter privado (incluyendo cientos de ellas de características marcadamente mercantiles, más que académicas). Esta tendencia se presenta también en los postgrados:

Las tendencias al crecimiento de la matrícula en las últimas décadas muestran que entre 1989-1990 y 1998-1999, la matrícula pasó de 45.900 a 111.200 estudiantes, superando las previsiones de los planes de expansión formulados por los gobiernos. El sector privado creció a tasas mayores que el público: mientras que hacia 1990, los postgrados privados comprendían el $19 \%$ de la matrícula, hacia el fin de la década representaban el 37\% (p. 179).

Esta tendencia se mantuvo en los años siguientes. En 2002, 60.3\% de los estudiantes de postgrado pertenecía a universidades públicas y $39.7 \%$ a privadas, y en $2005-2006,56.3 \%$ de los alumnos realizaba

12 En este marco han sido dos los principales programas oficiales de apoyo, no necesariamente bien coordinados entre sí, pero de creciente cobertura: el Programa de Mejoramiento del Profesorado (PROMEP) de la Secretaría de Educación Pública, y el Programa de Formación de Científicos y Tecnólogos del CONACyT. Entre ambos programas se da cuenta de "casi el 90\% del total de becas sostenidas por el gobierno federal” (Luchilo, 2010b, p. 108). 
estudios de postgrado en instituciones de educación superior públicas y $43.7 \%$ en privadas. El crecimiento de los postgrados de las universidades privadas se produjo sobre todo en las maestrías, donde las particulares representan $50.3 \%$ del total de la matrícula. En lo relativo al campo del conocimiento, la matrícula de las universidades particulares se concentra muy ampliamente en las ciencias sociales y administrativas. Así mismo, la matrícula de las universidades privadas está menos concentrada geográficamente (que la de las públicas) (Luchilo, 2010b).

La proliferación de programas de postgrado ha sido extraordinaria y se ha realizado casi sin control oficial. México pasó de 879 programas en 1980 a 5425 en 2005. Para el ciclo escolar 2010-2011, la ANUIES informaba de 6708 programas, con una matrícula de 40560 en especialidades, 144543 en maestrías y 23122 en doctorados, para un total de 208225 estudiantes de postgrado y un promedio de 31 por programa, cifra que parece extremadamente baja. Al mismo tiempo, a partir de 1991, el CONACyT y la Secretaría de Educación Pública (SEP) han operado un programa de evaluación, que se convirtió en la práctica en un sistema de acreditación de creciente rigor e influencia, denominado en la actualidad Programa Nacional de Postgrados de Calidad (PNPC), que establece como misión la de "fomentar la mejora continua y el aseguramiento de la calidad del postgrado nacional, que dé sustento al incremento de las capacidades científicas, tecnológicas, sociales, humanísticas, y de innovación del país" (CONACyT, 2013b). ${ }^{13}$ En el Marco de Referencia para la Evaluación y Seguimiento de Programas de Postgrado se establece que el PNPC "está conformado por dos vertientes":

1. El Padrón Nacional de Postgrados (PNP), integrado por dos niveles de calidad: I. Competencia internacional, II. Consolidados.

2. Programa de Fomento a la Calidad del Postgrado (PFCP), integrado por dos niveles de calidad: III. En Consolidación, IV. Reciente creación.

13 Al momento de mandar a impresión el presente artículo, el contenido de la dirección electrónica del CONACyT había sido modificado. Información equivalente puede encontrarse en http://www.conacyt.gob.mx/index.php/ el-conacyt/convocatorias-y-resultados-conacyt/convocatorias-pnpc/convocatorias-cerradas-pnpc/915-modalidad-escolarizada/file 
También se señala que:

Los campos de orientación de los programas académicos de postgrado son dos: los Programas de Postgrado con Orientación Profesional, que ofrecen los niveles de especialidad o de maestría, y los Programas de Postgrado con Orientación a la Investigación, que ofrecen los niveles de maestría o de doctorado.

\section{Y se declara lo siguiente:}

El ingreso de los programas de postgrado en el PNPC, representa un reconocimiento público a su calidad, con base en procesos de evaluación y seguimiento realizados por el comité de pares, por lo que el PNPC coadyuva al Sistema de Garantía de la Calidad de la educación superior. Asimismo, es un referente confiable acerca de la calidad de la oferta educativa en el ámbito del postgrado, que ayuda y orienta a los diferentes sectores del país, para que opten por los beneficios que otorga la formación de recursos humanos de alto nivel (CONACyT, 2013b).

El sistema de evaluación de los programas se basa en 6 categorías que constituyen los principales rubros de análisis que el programa debe satisfacer: a) Estructura del programa, b) Estudiantes, c) Personal académico, d) Infraestructura y servicios, e) Resultados y f) Cooperación con otros actores de la sociedad, y 19 "criterios que especifican estas categorías. ${ }^{14}$ Los parámetros de evaluación, según el nivel y la orientación de cada programa, imponen mínimos, en muchos de los indicadores de los criterios, para otorgar y sostener un determinado "nivel de calidad", definido finalmente por un comité de pares y el Consejo Nacional de Postgrado (CNP). De esa acreditación depende la posibilidad del programa de recibir apoyos oficiales, especialmente becas para sus

14 Los "criterios describen la tendencia de una actividad tipificada en el programa; permiten realizar el análisis de los procesos cognitivos, desde su planeación hasta el impacto en los resultados. Además son verificables, pertinentes y específicos, con capacidad orientadora y predictiva. Los criterios están formulados de manera esencialmente cualitativa, dejando amplios grados de libertad para su adecuación a la orientación y tipo de postgrado" (CONACyT, 2013b). 
estudiantes. La evaluación de los programas se realiza periódicamente, conforme al proceso resumido en la Figura 2.

En octubre de 2013, formaban parte del PNPC 1684 programas, 177 del nivel de especialidad, 980 de maestría y 527 de doctorado. De ese total, 333 programas $(19.7 \%)$ corresponden al Área de Ciencias Sociales (106 doctorados, 214 maestrías y 13 especialidades). Según el nivel, la categoría alcanzada y el régimen institucional, estos programas se distribuyen como se indica en la Tabla 2.

Cada vez más, una proporción creciente de estos programas adopta la denominación "Ciencias Sociales" u otras similares (como "Ciencias Políticas y Sociales" de la Universidad Nacional Autónoma de México [UNAM]), en vez de las denominaciones tradicionales de las disciplinas clásicas del área, para incorporar mediante la definición de "Líneas de Generación y Aplicación del Conocimiento", relaciones que pudieran llamarse interdisciplinarias o incluso transdisciplinarias en los procesos de formación de investigadores, así como para aprovechar mejor los recursos instalados que han debido crecer a una velocidad que pone en riesgo la calidad de su integración (Fuentes Navarro, 2012). Esta condición de la institucionalización de los altos estudios en ciencias sociales ha influido fuertemente en el desarrollo de ciertos campos, como el de los estudios de la comunicación en las universidades mexicanas, tal como puede apreciarse en el análisis de la siguiente sección.

\section{LA DESARTICULACIÓN MÚLTIPLE, SUS PERSPECTIVAS Y CONDICIONES DE FUTURO}

En la Tabla 3 se enlistan los 30 programas de postgrado (maestrías y doctorados) en los que se incluye formación en comunicación y que cuentan con acreditación vigente en octubre de 2013 por el PNPC, en alguno de sus cuatro niveles. Hay un aumento considerable, sobre todo de programas instalados en universidades públicas, respecto de los 19 programas incluidos en el recuento realizado en 2011 para la CONFIBERCOM, y que son adicionales a los programas no acreditados, cuyo número prácticamente no creció en el periodo, pues pasó de 62 en 2011, a 63 en 2013. La suma total de programas aumentó así, 


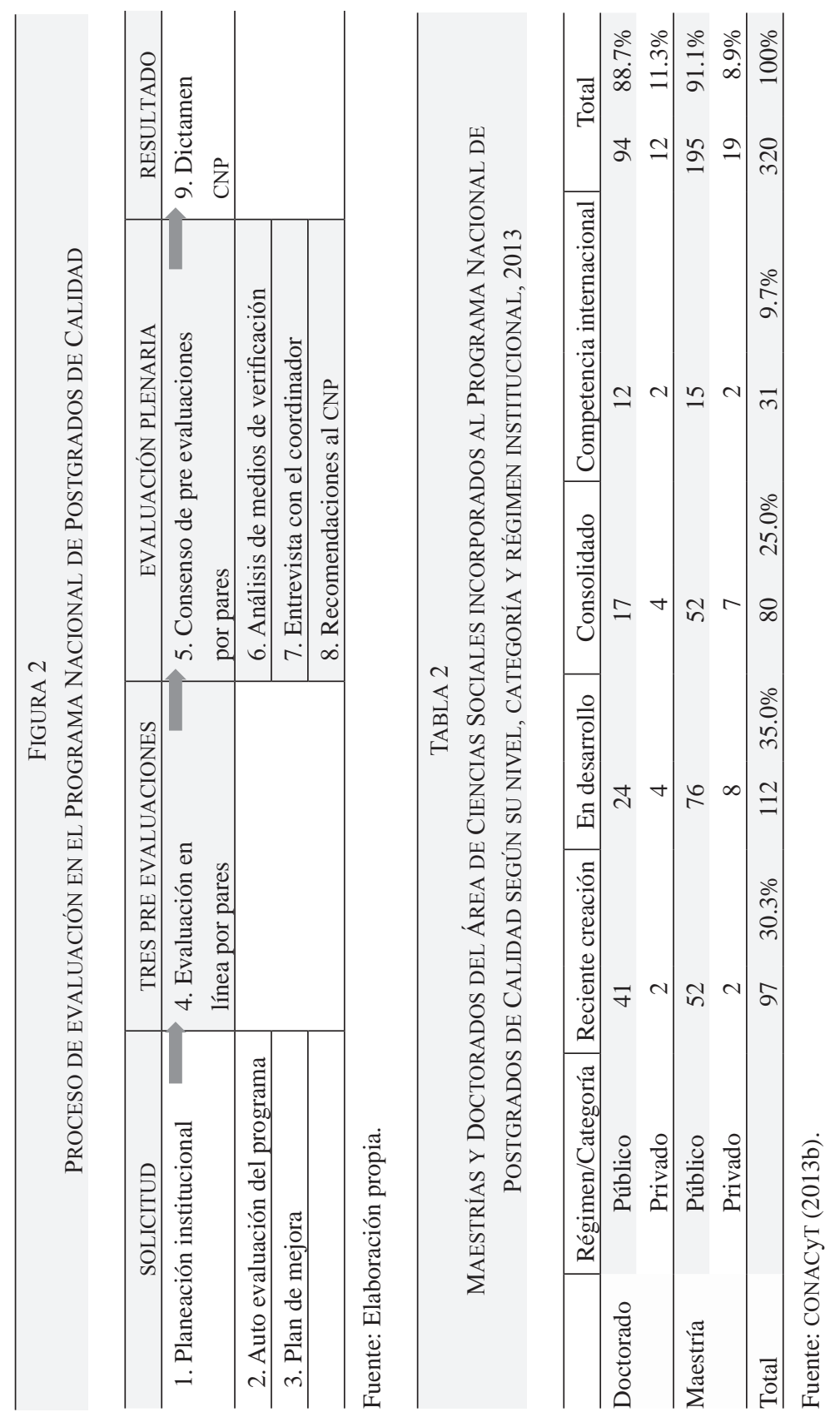


apenas, de 81 (72 maestrías y 9 doctorados) a 83 (67 maestrías y 16 doctorados); aunque se crearon algunos nuevos programas, también se cerraron otros, y además cambió la información disponible, por lo que cabe suponer ahora una confiabilidad mayor que en el informe 2011 de CONFIBERCOM. 15

Cabe subrayar que los 12 programas de doctorado y 6 de las maestrías de la Tabla 3 incluyen la formación en comunicación dentro de denominaciones interdisciplinarias más amplias: 100\% de los doctorados y un tercio de las maestrías, o en suma, $60 \%$ de los postgrados acreditados en el PNPC.

Pero la oferta de postgrados en comunicación en México es, como en las demás especialidades, mixta o dual: por una parte, algunas instituciones públicas y privadas tradicionales han conseguido consolidar, sobre todo mediante la conformación de plantas docentes de tiempo completo dedicadas a la investigación, programas de postgrado acreditables por el PNPC del CONACyT, 16 mientras que otras instituciones han optado por atender las demandas de especialización profesional (en buena medida impulsadas por el subempleo generado por el estancamiento económico imperante), mediante el modelo de postgrados de consumo (Lucio, 2002), que no requiere más que docentes de tiempo parcial y no incluye a la investigación académica entre sus insumos formativos.

En la Tabla 4 se enlistan los programas de postgrado en comunicación que completan la oferta nacional desde propuestas casi siempre predominantemente mercantiles (y casi nunca profesionalizantes en

15 Ese informe (Fuentes Navarro \& Bustamante Farias, 2012) se basó sobre todo en exploraciones mediante Internet. Ahora, pudo basarse la información en fuentes más confiables, pues tanto ANUIES como CONACyT mejoraron sus bases disponibles vía Internet, aunque la actualización de la primera deja todavía mucho que desear.

16 Son los casos, evidenciados en la Tabla 3, de los postgrados en comunicación acreditados que han sostenido instituciones privadas o particulares tradicionales como el Tec de Monterrey, la Universidad Iberoamericana o el Instituto Tecnológico y de Estudios Superiores de Occidente además de las principales universidades públicas del país. 


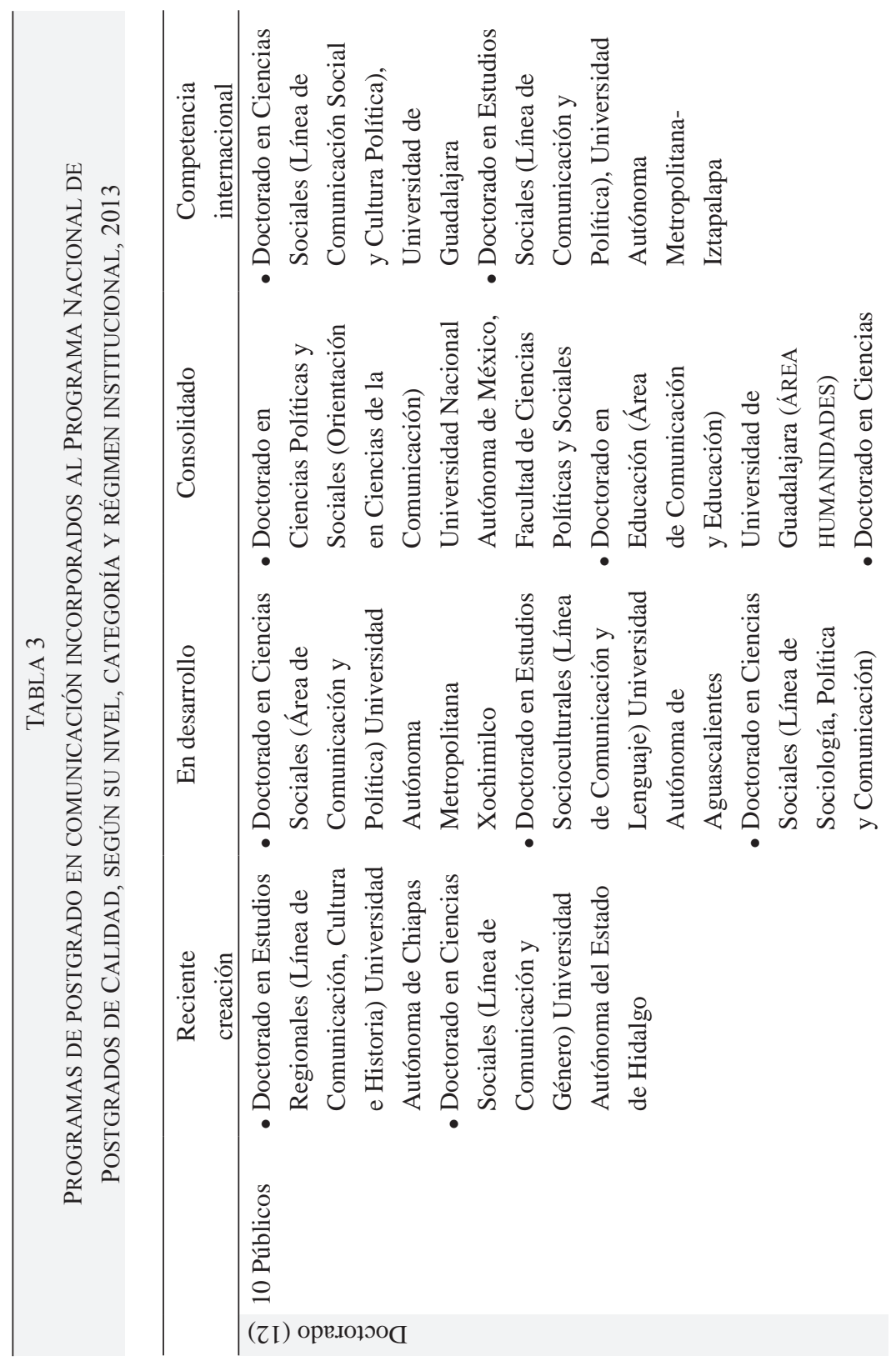




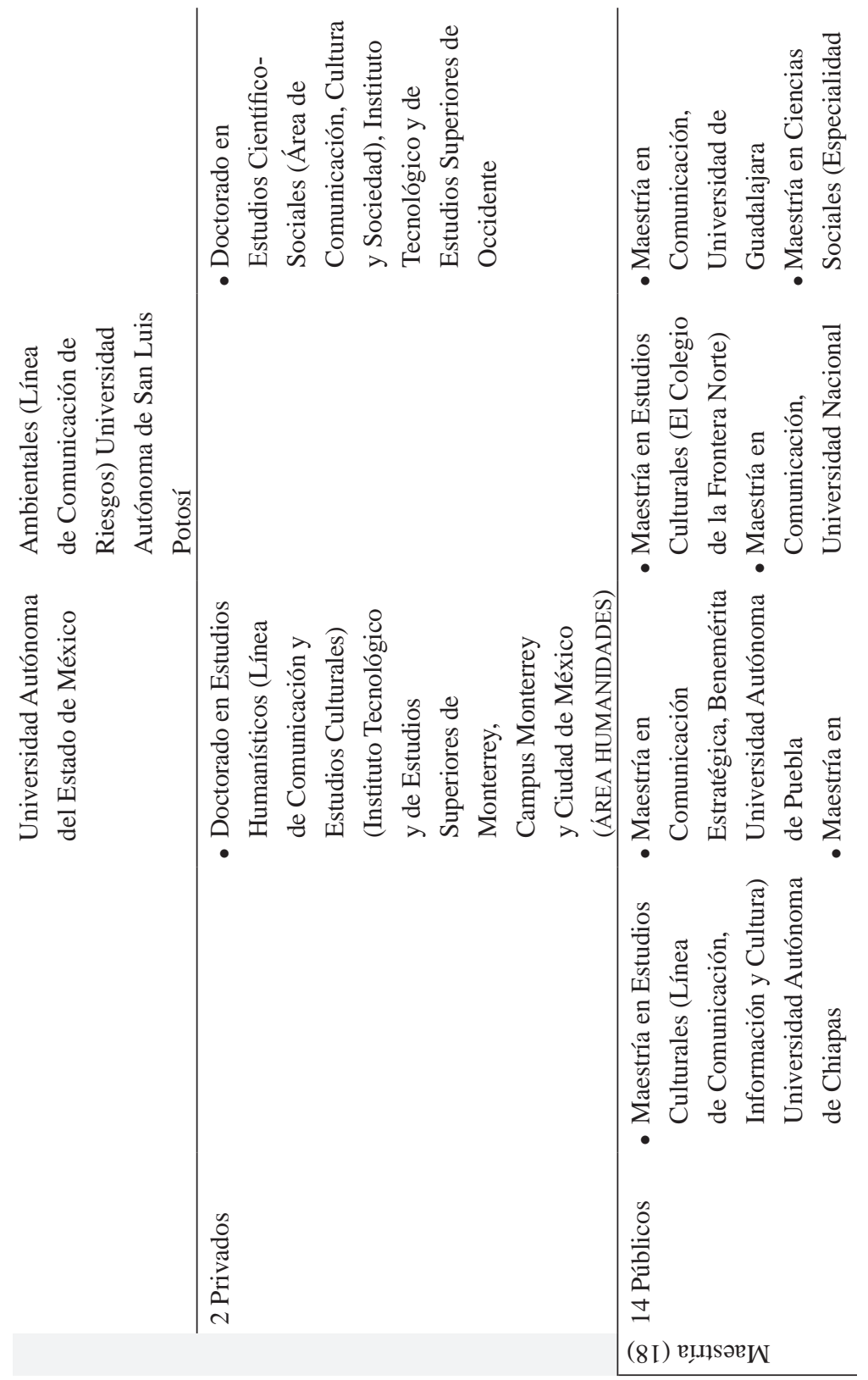




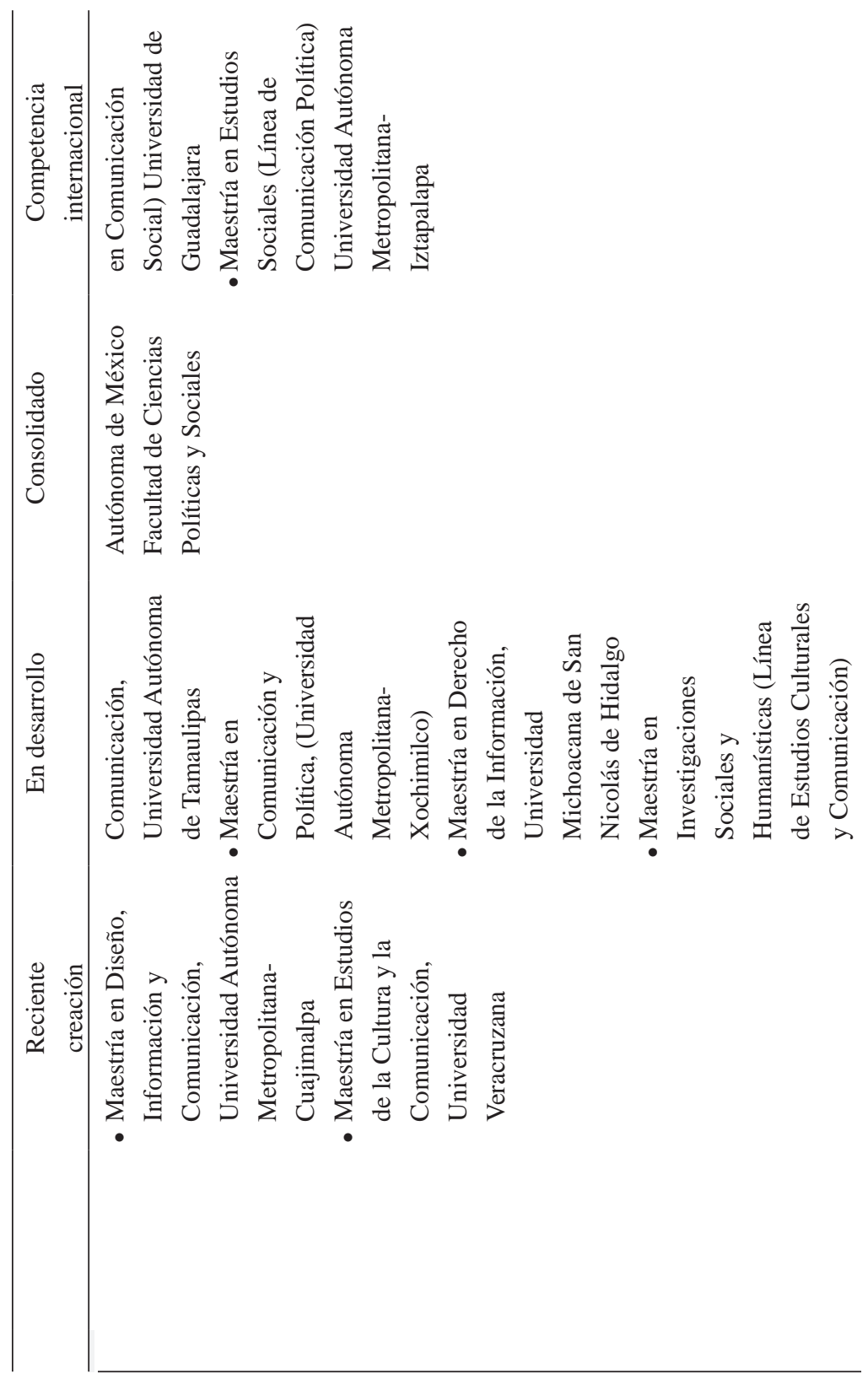




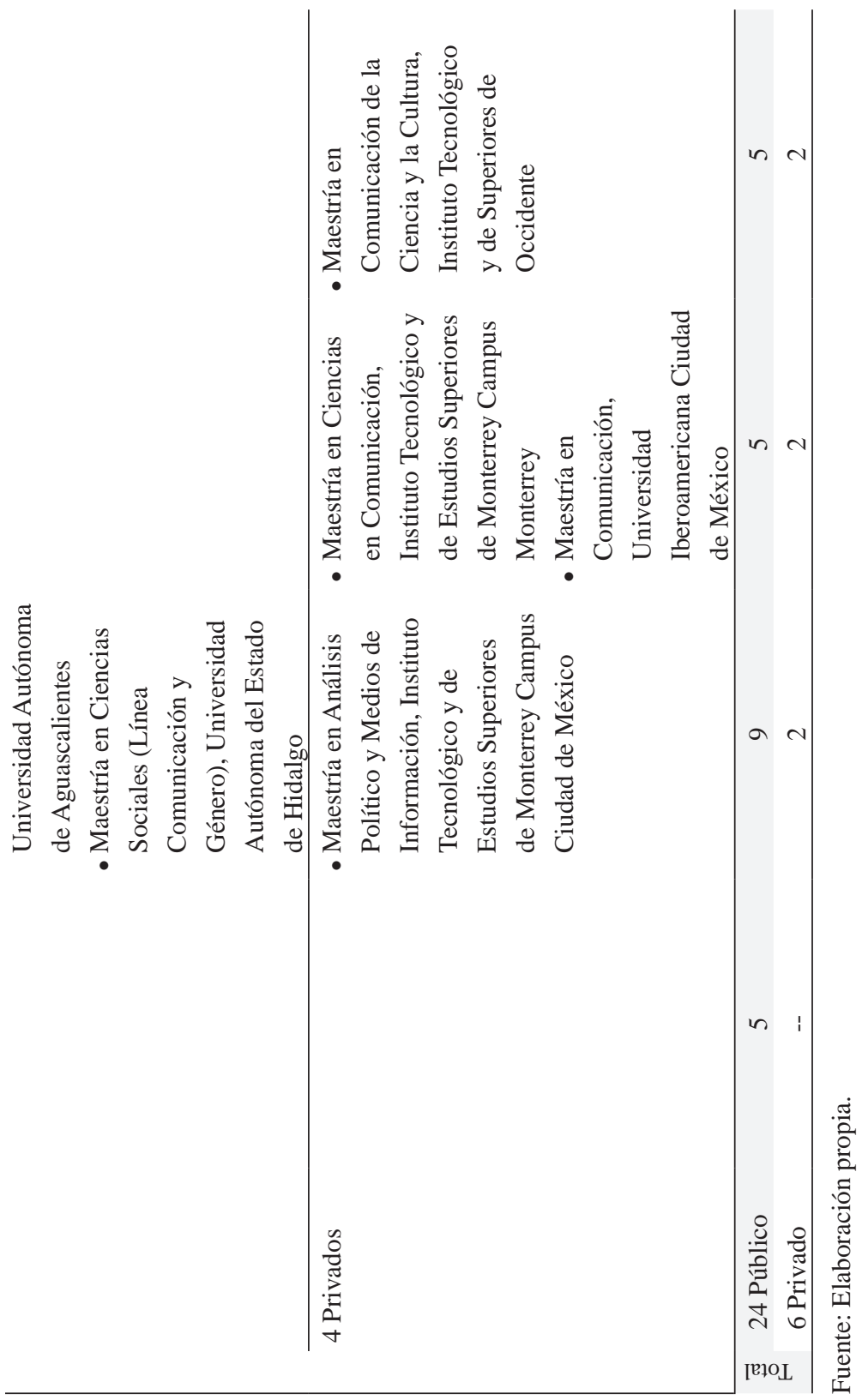




\section{TABLA 4}

PROGRAMAS DE POSTGRADO EN COMUNICACIÓN

CON RECONOCIMIENTO DE VALIDEZ OFICIAL DE ESTUDIOS PERO SIN

ACREDITACIÓN (PNPC), SEGÚN SU NIVEL Y RÉGIMEN INSTITUCIONAL, 2013

\begin{tabular}{|c|c|c|}
\hline & & Programa de Postgrado \\
\hline \multirow{3}{*}{ 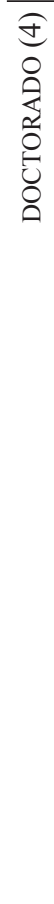 } & 0 Públicos & \\
\hline & 4 Privados & $\begin{array}{l}\text { - Doctorado en Comunicación y Pensamiento Estratégi- } \\
\text { co (Centro Avanzado de Comunicación Eulalio Ferrer) } \\
\text { (Ciudad de México) } \\
\text {-Doctorado en Estudios Transdisciplinarios de la Cultura } \\
\text { y la Comunicación (Instituto de Investigación en Comu- } \\
\text { nicación y Cultura) (Ciudad de México) } \\
\text { - Doctorado en Comunicación Aplicada (Universidad } \\
\text { Anáhuac del Norte) (Ciudad de México) } \\
\text {-Programa Internacional de Doctorado en Comunicación } \\
\text { (Universidad Iberoamericana Ciudad de México) (Ciu- } \\
\text { dad de México) }\end{array}$ \\
\hline & 5 Públicos & $\begin{array}{l}\text { - Maestría en Ciencias de la Comunicación (Instituto } \\
\text { Campechano) (Campeche) } \\
\text { - Maestría en Comunicación (Universidad Autónoma de } \\
\text { Baja California) } \\
\text { - Maestrías en Ciencias de la Comunicación y en Perio- } \\
\text { dismo (Universidad Autónoma de Chihuahua) } \\
\text { - Maestría en Ciencias de la Comunicación (Universidad } \\
\text { Autónoma de Nuevo León) }\end{array}$ \\
\hline 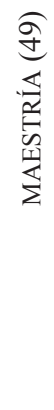 & 44 Privados & $\begin{array}{l}\text { - Maestrías en Comunicación Institucional, en Comu- } \\
\text { nicación Política, en Relaciones Públicas y en Publici- } \\
\text { dad (Centro Avanzado de Comunicación Eulalio Ferrer) } \\
\text { (Ciudad de México) } \\
\text { - Maestría en Comunicación y Mercadotecnia (Centro de } \\
\text { Estudios Universitarios) (Nuevo León) } \\
\text { - Maestría en Comunicación Periodística y sus Nuevas } \\
\text { Tecnologías (Centro de Estudios Universitarios en Perio- } \\
\text { dismo y Arte en Radio y Televisión) (Ciudad de México) }\end{array}$ \\
\hline
\end{tabular}




\begin{tabular}{|c|c|}
\hline & Programa de Postgrado \\
\hline \multirow{36}{*}{ 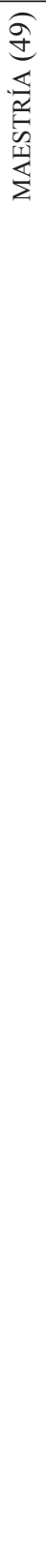 } & - Maestrías en Periodismo Económico y en Periodismo \\
\hline & Político (Escuela de Periodismo Carlos Septién García) \\
\hline & (Ciudad de México) \\
\hline & - Maestrías en Comunicación Pública y en Comunica- \\
\hline & ción y Medios Digitales (Fundación Universidad de Las \\
\hline & Américas Puebla) (Puebla) \\
\hline & -Maestrías en Comunicación y Estudios de la Cultura, en \\
\hline & Comunicación y Lenguajes Visuales y en Comunicación \\
\hline & y Medios Virtuales (Instituto de Investigación en Comu- \\
\hline & nicación y Cultura) (Ciudad de México) \\
\hline & - Maestría en Comunicación Organizacional (Instituto de \\
\hline & Estudios de la Comunicación de Yucatán) (Yucatán) \\
\hline & - Maestría en Tecnología Educativa (Instituto Latino- \\
\hline & americano de la Comunicación Educativa) (Ciudad de \\
\hline & México) \\
\hline & - Maestría en Comunicaciones Corporativas (Universi- \\
\hline & dad Anáhuac de Cancún) (Quintana Roo) \\
\hline & - Maestría en Comunicación Corporativa y Mercadotec- \\
\hline & nia (Universidad Anáhuac de Xalapa) (Veracruz) \\
\hline & - Maestría en Comunicación Estratégica e Imagen Ins- \\
\hline & titucional (Universidad Cuauhtémoc) (Aguascalientes) \\
\hline & - Maestría en Comunicación (Universidad de la Comuni- \\
\hline & cación ) (Ciudad de México) \\
\hline & - Maestrías en Comunicación Organizacional y en Co- \\
\hline & municación Social y Política (Universidad de La Salle \\
\hline & Bajío) (Guanajuato) \\
\hline & -Maestría en Relaciones Públicas (Universidad de León) \\
\hline & (Guanajuato) \\
\hline & - Maestría en Cibercomunicación (Universidad del Alti- \\
\hline & plano) (Tlaxcala) \\
\hline & - Maestría en Comunicación Organizacional (Universi- \\
\hline & dad del Ciudad de México) (Ciudad de México) \\
\hline & - Maestría en Comunicaciones Corporativas (Universi- \\
\hline & dad del Mayab -Anáhuac) (Yucatán) \\
\hline & - Maestría en Comunicación y Estrategias Publicitarias \\
\hline & (Universidad del Sol) (Morelos) \\
\hline
\end{tabular}




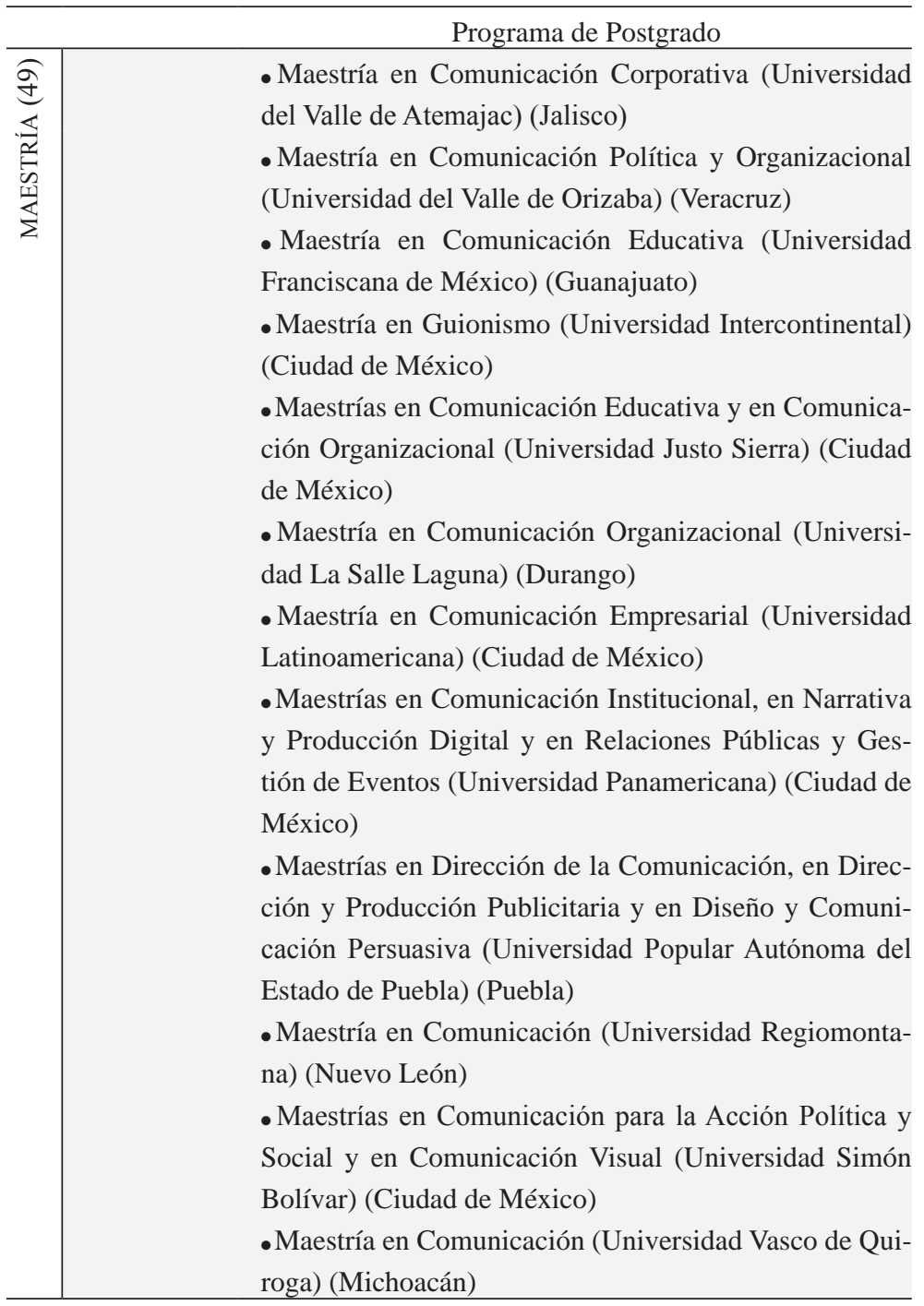

Fuente: Elaboración propia. 
sentido estricto), que en casi ningún caso superan una antigüedad de 10 años, y que se diferencian casi todos, cada vez más, de los programas acreditados que aparecen en la Tabla 3, aunque entre ellos hay programas impartidos en instituciones (públicas y privadas) de indudable solidez académica.

Tanto entre los acreditados como entre los no acreditados, la propia historia de cada uno de los programas expresa la conjunción de factores contextuales y estructurales, entre los que hay que incluir sin duda la multivalencia epistémica de la comunicación y la desarticulación múltiple que ha caracterizado a la institucionalización académica de su estudio universitario (Fuentes Navarro, 1992, 1995, 2005). De ahí surge, en parte, la disyunción o divergencia creciente entre los dos modelos de postgrado en comunicación que se pueden identificar en México (y también en otros países): el postgrado académico orientado en y hacia la investigación, y el postgrado profesional orientado por el mercado. Cabe hacer notar, al respecto, la coincidencia con la ambivalencia del grado de maestría, incómodamente intermedio entre la formación universitaria de profesionales en las licenciaturas y la formación universitaria de investigadores en los doctorados, por lo que se hace más necesario aún problematizar e identificar las articulaciones estratégicas operantes entre las disciplinas y las profesiones.

El hecho de que solo muy recientemente se intenten establecer en México programas de doctorado denominados en comunicación ${ }^{17}$ quizá sea ilustrativo de la disyunción mencionada, pues aun con bases en la investigación, varios de ellos parecen orientarse hacia el polo profesionalizante. La profesionalización avanzada de investigadores en comunicación, durante al menos las últimas cuatro décadas, ha dependido en

17 En un caso, "Aplicada" (Universidad Anáhuac), en otro asociado al "Desarrollo Estratégico" (Centro Avanzado de Comunicación Eulalio Ferrer) en un tercero a los "Estudios Transdisciplinarios de la Cultura" (Instituto de Investigación en Comunicación y Cultura) y en el más reciente a un "programa internacional" (Universidad Iberoamericana) en referencia a un convenio de colaboración firmado al respecto con el Instituto Tecnológico y de Estudios Superiores de Occidente, la Universidad Javeriana de Bogotá, la Universidad de Lima y la Autónoma de Barcelona. 
buena medida de la formación doctoral en el extranjero o en programas no relacionados exclusiva o siquiera prioritariamente con este campo. Los espacios específicos (como Áreas de Concentración o Líneas de Generación y Aplicación del Conocimiento) que se han abierto en programas acreditados en el PNPC, integran la formación de investigadores de la comunicación en entornos "inter" o "multidisciplinarios" de denominación más amplia (véase Tabla 3), en muchos casos con ventaja sobre una formación más estrechamente "disciplinaria", aunque ciertamente con una visibilidad más bien precaria.

De cualquier manera, la demanda por estudios de postgrado en comunicación en México presenta escalas y condiciones muy diferentes de las licenciaturas. Aunque no se dispone de cifras oficiales completas, puede calcularse que con muy pocas excepciones, la matrícula de los programas profesionalizantes no acreditados es muy escasa, y su eficiencia terminal ínfima, pues los mercados de trabajo no suelen apreciar ni exigir títulos académicos. Por su parte, la matrícula de los programas acreditados por el PNPC es limitada también, debido principalmente a la exigencia de dedicación de tiempo completo a los estudios que supone el otorgamiento de becas de manutención con las que CONACyT apoya a los estudiantes inscritos en programas reconocidos por el PNPC, a la escasa formación inicial para la investigación en las licenciaturas, y a los rigurosos procesos de selección de estudiantes que los programas acreditados deben establecer como parte de sus indicadores de calidad.

También cabe hacer notar que una alta y al parecer creciente proporción (variable según la universidad) de los estudiantes de postgrado en comunicación en los programas acreditados cuenta con estudios previos en otras disciplinas, y también que el porcentaje de estudiantes extranjeros inscritos en estos programas alcanza hasta 20\% del total en algún caso. En consecuencia, hay una creciente desarticulación entre niveles de formación, entre especialización y fundamentación teóricometodológica, entre investigación y docencia y, en último término, entre "el conocimiento y la acción social" (Fuentes Navarro, 1992, p. 24), con la consecuencia, entre otras, de una creciente confusión entre campos, disciplinas y profesiones en la institucionalización de los estudios 
sobre la comunicación en las universidades (Fuentes Navarro, 1995). Se puede retomar aquí, respecto de los postgrados, una consideración que años atrás se propuso para analizar los estudios de comunicación en su nivel de licenciatura:

Con el énfasis que se entiendan, y al igual que casi cualquier otra área de profesionalización universitaria, [estos programas] están sujetos necesariamente a una relación constitutiva, y difícil de articular, con otras disciplinas (académicas) y especialidades (profesionales). Si ha sido difícil en todas partes encontrar y mantener las articulaciones más productivas en términos de aprendizaje en las zonas curriculares básicas de los programas de comunicación, más difícil tiende a ser esta articulación en las zonas de "especialización" profesional, debido sobre todo a que, con variantes notables, la especificidad de los múltiples campos profesionales asociables con la carrera de comunicación es muy débil y cambiante.

En un plano más amplio de análisis, puede también afirmarse que, especialmente en la última década, como causa y efecto de múltiples factores, la identidad disciplinaria de los estudios de comunicación, sobre todo en lo que respecta a la investigación y los postgrados, se ha vuelto mucho más compleja de sostener y es objeto de debate no sólo teórico e intelectual, sino también estratégico en los planos institucional, político y profesional. Los procesos de institucionalización, social y cognoscitiva, del campo académico de la comunicación en México y América Latina se han desarrollado manteniendo y reforzando una desarticulación múltiple, que a los problemas "importados" de Estados Unidos y Europa suma problemas muy propios. El principal es confundir, como lo ha sintetizado Martín-Barbero (2001), las condiciones de desarrollo del o los mercados profesionales, con las exigencias de un campo intelectual (Fuentes Navarro, 2005, p. 37).

Desde otro ángulo, la situación refleja también lo que afirmaba hace dos décadas el académico estadounidense David Swanson (1993):

Reconocemos a la comunicación como un campo interdisciplinario compuesto por diversos subcampos que están conectados histórica y políticamente, por la organización de los programas, pero no intelectualmente. A un 
nivel global, esos subcampos comparten un interés por la "comunicación", cada uno en sus propios términos y para sus propios propósitos, pero sería engañoso suponer que ese interés compartido tenga contenido sustantivo y aporte una perspectiva disciplinaria común (p. 411).

Para el futuro inmediato, en el espacio institucional que ocupan los estudios de postgrado en comunicación en México, no parece haber elementos suficientes aún para determinar si la desarticulación múltiple habrá de desembocar en una configuración aparentemente paradójica: la del estancamiento concentrado, es decir, la dificultad para producir de otras maneras que no sean las inerciales en las mismas pocas instituciones de siempre, o en la evolución postdisciplinaria que consolide un modo de producción académica orientado por la identificación y formulación de problemas en el entorno sociocultural (Menand, 2010), antes que por la prescripción de fórmulas teórico-metodológicas (disciplinarias e interdisciplinarias), cuya extrapolación y aplicación a contextos y situaciones diversos de aquellos respecto de los cuales se originaron, se muestran crecientemente impertinentes e ineficaces.

\section{Bibliografía}

Abbott, A. (1988). The system of professions. An essay on the division of expert labor. Chicago, IL, EE.UU.: The University of Chicago Press. Abbott, A. (2001). The chaos of disciplines. Chicago, IL, EE.UU.: The University of Chicago Press.

Albornoz, M. (Ed.). (2010). El estado de la ciencia. Principales indicadores de Ciencia y Tecnología Iberoamericanos/Interamericanos. Buenos Aires, Argentina: Redes-Centro de Estudios sobre Ciencia, Desarrollo y Educación Superior.

Asociación Nacional de Universidades e Instituciones de Educación Superior-ANUIES. (2011). Anuario Estadístico de Educación Superior. Recuperado el 29 de diciembre de 2013 de http://www.Aniues. $\mathrm{mx} /$ content.php?varSectionID $=166$

Boromisza-Habashi, D. (2013). Which way is forward in communication theorizing? An interview with Robert T. Craig. Communication Theory, 23, 417-432. 
Cámara de Diputados. (2013). Ley de Ciencia y Tecnología. Recuperado el 30 de enero de 2014 de http://www.diputados.gob.mx/LeyesBiblio/pdf/242.pdf

Cimadevilla, G. \& García Corredor, C. (2012). Posgrados en Comunicación en América del Sur: heterogeneidades entrelazadas. En M. I. Vassallo de Lopes (Coord.), Posgrados en comunicación en Iberoamérica: políticas nacionales e internacionales (pp. 49-83). São Paulo, Brasil: Confederación Iberoamericana de Asociaciones Científicas y Académicas de Comunicación/Universidad de São Paulo-Programa de Posgrado en Ciencias de la Comunicación.

Consejo Nacional de Ciencia y Tecnología-CONACyT. (2010). Informe General del Estado de la Ciencia y Tecnología, México 2009. Recuperado el 26 de septiembre de 2011 de http://siicyt.gob.mx/siicyt/ docs/contenido/IGECYT_2009.pdf

Consejo Nacional de Ciencia y Tecnología-CONACyT (2013a). Reglamento del Sistema Nacional de Investigadores. Recuperado el 30 de enero de 2014 de http://www.conacyt.gob.mx/ElConacyt/Documentos\%20Normatividad/Reglamento_del_SNI.pdf

Consejo Nacional de Ciencia y Tecnología-CONACyT. (2013b). Sistema de Consultas del Programa Nacional de Posgrados de Calidad. Recuperado el 30 de enero de 2014 de http://surtmp.main.conacyt. $\mathrm{mx} /$ consultasPNPC/inicio.php

Consejo Nacional de Ciencia y Tecnología-CONACyT. (2014). Programa Nacional de Posgrados de Calidad PNPC-2. Marco de referencia para la evaluación y seguimiento de programas de posgrado. Recuperado el 30 de enero de 2014 de http://www.conacyt.gob.mx/ index.php/el-conacyt/convocatorias-y-resultados-conacyt/convocatorias-cerradas-pncp/915-modalidad-escolarizada/file

Cruz Cardona, V. \& Martos Perales, F. (2010). Evaluación de la calidad de los programas de maestría y doctorado en Iberoamérica. Revista Digital Universitaria, 11(5). Recuperado el 15 de diciembre de 2013 de http://www.revista.unam.mx/vol.11/num5/art45/art45.pdf

De la Peza Casares, M. C. (2013). Los estudios de comunicación: disciplina o indisciplina. Comunicación y Sociedad, 20, 11-32.

De Moragas i Spà, M. (2011). Interpretar la comunicación: estudios sobre medios en América y Europa. Barcelona, España: Gedisa. 
Federación Latinoamericana de Facultades de Comunicación SocialFELAFACS. (2009). Informe final del proyecto: Mapa de los centros y programas de formación en Comunicación y Periodismo en América Latina y el Caribe. Lima, Perú: FELAFACS/Organización de las Naciones Unidad para la Educación, la Ciencia y la Cultura.

Foro Consultivo Científico y Tecnológico-FCCYT. (2013). Sistema Nacional de Investigadores. Recuperado el 30 de enero de 2014 de http://www.foroconsultivo.org.mx/documentos/acertadístico/conacyt/sistema_nacional_de_investigadores.pdf

Fuentes Navarro, R. (1992). El estudio de la comunicación desde una perspectiva sociocultural en América Latina. Diálogos de la Comunicación, 32, 16-26.

Fuentes Navarro, R. (1995). La institucionalización académica de las ciencias de la comunicación: campos, disciplinas, profesiones. En J. Galindo \& C. Luna (Coords.), Campo académico de la comunicación. Hacia una reconstrucción reflexiva (pp.45-78). Guadalajara, México: Instituto Tecnológico y de Estudios Superiores de Occidente/Consejo Nacional para la Cultura y las Artes.

Fuentes Navarro, R. (2005). La configuración de la oferta nacional de estudios superiores en comunicación. Reflexiones analíticas y contextuales. En J. Calles (Ed.), Anuario CONEICC de Investigación de la Comunicación, XII (pp. 15-40). México: Consejo Nacional para la Enseñanza y la Investigación de las Ciencias de la Comunicación.

Fuentes Navarro, R. (2010). Maestrías en comunicación en México: análisis comparativo de sus perfiles de producción y reproducción académica. Comunicación y Sociedad, 14, 13-48.

Fuentes Navarro, R. (2011). Condiciones institucionales para la práctica de la investigación académica de la comunicación: la persistencia de la triple marginalidad en México. En E. Sánchez Ruiz (Coord.), Qué pasa con el estudio de los medios. Diálogo con las ciencias sociales en Iberoamérica (pp. 15-55). Zamora, España: Comunicación Social.

Fuentes Navarro, R. (2012). La formación de investigadores en ciencias sociales y humanas: agencia y estructura socioeducativa en la periferia de la sociedad del conocimiento. Signo y Pensamiento, 60, $62-72$. 
Fuentes Navarro, R. \& Bustamante Farías, O. (2012). La oferta académica de posgrados en Comunicación en México, Centroamérica y el Caribe: una expansión asimétrica y desarticulada. En M. Vassallo de Lopes (Coord.), Posgrados en comunicación en Iberoamérica: políticas nacionales e internacionales (pp.111-162). São Paulo, Brasil: Confederación Iberoamericana de Asociaciones Científicas y Académicas de Comunicación/ Universidad de São Paulo-Programa de Posgrados en Ciencias de la Comunicación.

Kunsch, M. K. (Org.). (2013). La comunicación en Iberoamérica: políticas científicas y tecnológicas, posgrado y difusión de conocimiento. Quito, Ecuador: Confederación Iberoamericana de Asociaciones Científicas y Académicas de Comunicación/Centro Internacional de Estudios Superiores de Comunicación para América Latina.

Kunsch, M. K. \& Marques de Melo, J. (Orgs.). (2012). Comunicação Ibero-americana. Sistemas midiáticos, diversidade cultural, pesquisa e pós-graduação. São Paulo, Brasil: Confederación Iberoamericana de Asociaciones Científicas y Académicas de Comunicación/ Universidad de São Paulo-Escuela de Comunicación y Artes/Federación Brasileña de la Ciencia y Comunicación Científica.

Luchilo, L. (Comp.). (2010a). Formación de posgrado en América Latina: políticas de apoyo, resultados e impactos. Buenos Aires, Argentina: Editorial Universitaria de Buenos Aires.

Luchilo, L. (2010b). México: tendencias e impactos de los programas de formación de posgrado. En L. Luchilo (Comp.), Formación de posgrado en América Latina: políticas de apoyo, resultados e impactos (pp.177-215). Buenos Aires, Argentina: Editorial Universitaria de Buenos Aires.

Lucio, R. (2002). Políticas de posgrado en América Latina. En R. Kent (Comp.), Los temas críticos de la educación superior en los años noventa (pp. 325-374). México: Facultad Latinoamericana de Ciencias Sociales/Universidad Autónoma de Aguascalientes/Fondo de Cultura Económica.

Marques de Melo, J. (2012). Prólogo. En M. Kunsch \& J. Marques de Melo (Orgs.), Comunicação Ibero-americana. Sistemas midiáticos, diversidade cultural, pesquisa e pós-graduação (pp. 9-12). São Paulo, Brasil: Confederación Iberoamericana de Asociaciones 
Científicas y Académicas de Comunicación/Universidad de São Paulo-Escuela de Comunicación y Artes/Federación Brasileña de la Ciencia y la Comunicación Científica.

Martín-Barbero, J. (2001). Deconstrucción de la crítica: nuevos itinerarios de la investigación. En M. I. Vassallo de Lopes \& R. Fuentes Navarro (Comps.), Comunicación, campo y objeto de estudio. Perspectivas reflexivas latinoamericanas (pp.15-42). Guadalajara, México: Instituto Tecnológico y de Estudios Superiores de Occidente/ Universidad Autónoma de Aguascalientes/Universidad de Colima/ Universidad de Guadalajara.

Mattelart, A. (1998). La mundialización de la comunicación. Barcelona, España: Paidós Ibérica.

Mattelart, A. (2006). Diversidad cultural y mundialización. Barcelona, España: Paidós Ibérica.

Menand, L. (2010). The marketplace of ideas. Reform and resistance in the American University. Nueva York, EE.UU.: W. Norton \& Company.

Organización de las Naciones Unidas para la Educación la Ciencia y la Cultura-UNESCO \& Consejo Internacional de Ciencias SocialesCICS. (2012). Informe sobre las ciencias sociales en el mundo, 2010. Las brechas del conocimiento. México: Autor/Foro Consultivo Científico y Tecnológico/Consejo Mexicano de Ciencias Sociales.

Rebeil Corella, M. A. (2009). México: fragmentación de la oferta educativa. En FELAFACS, Mapa de los centros y programas de formación en Comunicación y Periodismo en América Latina y el Caribe (pp. 69-87). Lima, Perú: FELAFACS/UNESCO.

Ruiz Gutiérrez, R. (2009). Tendencias y perspectivas de la educación superior, la ciencia, la tecnología y la innovación en México. Recuperado el 26 de septiembre de 2011 de http://www.amc.unam.mx/ Tendencias.pdf

Sierra Caballero, F. (2013). Hacia una epistemología del Sur: comunicología latina y agenda de investigación. En M. Kunsch (Org.), La comunicación en Iberoamérica: políticas cientificas y tecnológicas, posgrado y difusión de conocimiento (pp. 25-46). Quito, Ecuador: Confederación Iberoamericana de Asociaciones Científicas y Aca- 
démicas de Comunicación/Centro Internacional de Estudios Superiores de Comunicación para América Latina.

Swanson, D. L. (1993). Fragmentation, the field, and the future. Journal of Communication, 43(4), 163-173.

Trinidade, H. (Coord.). (2007). Las ciencias sociales en América Latina en perspectiva comparada. México: Siglo XXI.

Vassallo de Lopes, M. I. (Coord). (2012). Posgrados en comunicación en Iberoamérica: políticas nacionales e internacionales. São Paulo, Brasil: Confederación Iberoamericana de Asociaciones Científicas y Académicas de Comunicación/Universidad de São Paulo-Programa de Posgrado en Ciencias de la Comunicación.

Fecha de recepción: 06/02/14. Aceptación: 12/03/14. 